\title{
Envejecimiento y salud mental en contextos migratorios
}

\section{Ageing and mental health in the context of migration}

\section{Envelhecimento e saúde mental em contextos migratórios}

\author{
E. Torres-Hernández ${ }^{\mathrm{a}^{*}}$, B. Arias-López \\ ORCID: \\ ${ }^{a} 0000-0001-9273-1035$ \\ b 0000-0002-3326-0402 \\ Facultad de Enfermería de la Universidad de Antioquia, Medellín Colombia. \\ Recibido: 8 junio 2018 \\ Aceptado: 27 octubre 2018
}

\section{Resumen}

Introducción: La investigación en vejez y envejecimiento se ha vuelto prioritaria ante los procesos de transformación demográfica a nivel mundial, con la inversión de la pirámide y las consecuencias que ello conlleva, en contextos sociales cada vez más complejos; uno de estos escenarios es el contexto migratorio, el cual plantea retos y desafíos en su vida cotidiana que permiten la construcción de ciertas estrategias de vida, durante el proceso de envejecimiento.

El objetivo: Se enmarca en presentar una revisión narrativa sobre el estado de discusión actual acerca del envejecimiento y la salud mental en contextos migratorios, desde lo generado por distintas disciplinas que contribuyen al desarrollo del conocimiento de la disciplina enfermera.

Desarrollo: La lógica de presentación de esta revisión, parte de la diferenciación entre vejez y envejecimiento, los enfoques a partir de los cuales se ha analizado éste último y cómo se entrelaza el envejecimiento con dinámicas sociales como la migración, para finalmente presentar la discusión acerca de los abordajes de la salud mental, muy relevante en este tipo de situaciones.

Conclusión: Esta revisión buscó despertar el interés de la disciplina enfermera por la comprensión de la forma de vida de las personas mayores en diferentes situaciones contextuales, como la migración, que permitan generar un acercamiento a la generación de estrategias de cuidado cultural y socialmente competentes.

Palabras clave: Envejecimiento; vejez; salud mental; bienestar; inmigración; enfermería; México. 


\section{Abstract}

Introduction: Considering the current inverted population pyramid and its related consequences, researching on aging and the old age has become a priority in the analysis of the demographic transformation processes worldwide, particularly in the evermore complex social contexts we live in. One on these scenarios is the migration context, which bears additional challenges to the pursuit of life strategies to coping with the aging process.

Objective: This is a narrative review on the current state of discussion about aging and mental health and framed within the migration context while considering the diverse disciplines which contribute to the knowledge of nursing.

Development:The focusses on the differentiation and analysis between the old age and the aging process and how these are intertwined with social dynamics such as migration, are first addressed to finally present a discussion on the approaches to the related mental health issues.

Conclusion: This review contributes to the understanding on the coping life strategies of old people in different contexts, such as migration, so that the corresponding culturally and socially competent care plans can be developed.

Keywords: Aging; old age; mental health; wellbeing; migration; nursing; Mexico.

\section{Resumo}

Introdução: A pesquisa em idosos e idosos tem se tornado uma prioridade nos processos de transformação demográfica mundial, com a inversão da pirâmide e as conseqüências que isso acarreta, em contextos sociais cada vez mais complexos; Um desses cenários é o contexto migratório, que coloca desafios e desafios em seu cotidiano que permitem a construção de determinadas estratégias de vida durante o processo de envelhecimento.

O objetivo: enquadra-se em apresentar uma revisão narrativa sobre o estado da discussão atual sobre envelhecimento e saúde mental em contextos migratórios, a partir do gerado por diferentes disciplinas que contribuem para o desenvolvimento do conhecimento da disciplina de enfermagem.

Desenvolvimento: A lógica de apresentação desta revisão, parte da diferenciação entre velhice e envelhecimento, as abordagens a partir das quais esta última tem sido analisada e como o envelhecimento está ligado a dinâmicas sociais como a migração, para finalmente apresentar a discussão sobre de abordagens de saúde mental, muito relevante neste tipo de situação.

Conclusão: Esta revisão buscou despertar o interesse da disciplina de enfermagem para a compreensão do modo de vida dos idosos em diferentes situações contextuais, como a migração, que permitem gerar uma abordagem para a geração de estratégias de cuidado cultural e socialmente competentes.

Palavras chave: Envelhecimento; velhice; saúde mental; bem-estar; imigração; enfermagem; México.

\section{Introducción}

El cuidado a la salud de los adultos mayores ha tomado un lugar relevante para la enfermería y otras disciplinas en los últimos años, dado el incremento de este grupo poblacional a nivel mundial, y las complejas condiciones sociales que enfrentan. Particularmente, en contextos como el mexicano, una de estas situaciones es la cuestión migratoria, evento que sobresale dada su cercanía fronteriza con Estados Unidos, lugar privilegiado de recepción de migrantes, que genera una manera particular de enfrentar el proceso de envejecimiento para las personas mayores que se quedan en sus lugares de origen, cuyas redes familiares han migrado. Esta situación impone desafíos para su salud mental, por tanto deben generar estrategias de respuesta ante una nueva forma de vida, en ausencia de sus familiares, que les permita transitar y resolver su cotidianidad.

La triada envejecimiento, salud mental y migración, ha sido poco explorada por la disciplina de Enfermería, especialmente en el contexto mexicano, por lo cual se torna relevante reconocerla como un campo problemático que guarda relación con el bienestar y por ende con el cuidado de la vida del 
adulto mayor, convirtiéndose en punto central para la práctica profesional y el desarrollo disciplinar en enfermería. Con este referente, el objetivo es presentar una revisión narrativa de lo producido y las tendencias en relación con el envejecimiento y la salud mental en contextos migratorios, desde distintos campos disciplinares, que puedan aportar a la disciplina de enfermería desde lo conceptual pero también desde lo práctico.

\section{Método}

Revisión narrativa derivada del proyecto de tesis doctoral en curso titulada: Los que se quedan... salud mental de adultos mayores inmersos en contextos de alta migración en México.

Este tipo de revisión tiene como finalidad identificar, analizar, valorar e interpretar el cuerpo de conocimientos sobre un tema y permite tener una información más amplia sobre el mismo, de tal manera que favorezca la revisión del estado del arte o la identificación de vacíos en el conocimiento del tema en particular ${ }^{1}$.

Para la construcción de la presente revisión se consideró la propuesta de Silamani ${ }^{1}$. Para ello, se inició por identificar como tema central la triada envejecimiento, salud mental y migración; posteriormente se seleccionó material bibliográfico producido y publicado en revistas indexadas; para la interpretación de resultados se procedió con la elaboración de fichas analíticas y matrices de análisis en donde se rescataron las principales corrientes teóricas, conceptos e ideas clave; el origen y definición de los temas; los campos epistemológicos y ontológicos; cuestiones principales y controversiales y los problemas que se han investigado al respecto, para ubicar de manera general las tendencias de la producción científica publicada.

Para la búsqueda de material bibliográfico, se generó un sondeo de las generalidades del tema para dar pie a la segunda búsqueda más minuciosa que permitiera la identificación de otros descriptores que favorecieran una focalización más precisa de lo producido. En la primera búsqueda se tomaron Descriptores en Ciencias de la Salud (DECS) en inglés y español, como envejecimiento, vejez, vejez activa, adulto mayor, viejo, persona mayor, envejecimiento activo, salud mental, funcionalidad; los cuales fueron rastreados en bases de datos como Scielo, Biblioteca Virtual en Salud (BVS), CUIDEN, Science Direct, Pub Med/Med Line, EBSCO, Dialnet; se eligieron estas fuentes por poseer producción científica de países de América Latina y el Caribe en ciencias de la salud, áreas biomédicas y cuidados de enfermería en Iberoamérica; la acotación temporal establecida fue del año 2006 al 2016, con la finalidad de ubicar los resultados más actuales con respecto al tema, la búsqueda se refinó a partir del uso del operador booleano AND, se realizaron diversas combinaciones; para identificar un total de 126 artículos en la primer búsqueda.

De los hallazgos en esta primera etapa, se pudieron identificar otras palabras claves relacionadas al tema de interés, como inmigración, afrontamiento, sufrimiento, emociones, bienestar; se trabajó en las mismas bases de datos anteriormente mencionadas y se mantuvo el filtro de temporalidad, idiomas de búsqueda y combinaciones con ayuda del operador booleano “AND”, en total quedaron 114 artículos en esta segunda búsqueda.

De los 240 artículos identificados, se seleccionaron 82 que explicitaban elementos combinados de la triada envejecimiento/vejez, salud mental y migración; se elaboró una matriz de análisis en donde se agruparon trabajos en relación con algunas duplas de interés. En concordancia con la dupla salud mental y envejecimiento, se encontraron investigaciones que emplearon principalmente diseños cuantitativos, Brasil fue el lugar de procedencia de la mayoría de los trabajos, de manera especial, en el campo de la psicología. Con la dupla migración y envejecimiento, el diseño predominante siguió también la lógica cuantitativa predominante en México y Estados Unidos, desde el campo de la sociología. De la revisión de estos productos se procedió a establecer las tendencias en la investigación y los vacíos del conocimiento en términos de poca claridad del tema y/o necesidad de ampliar o profundizar al respecto, que 
fueran relevantes para el conocimiento y la práctica del cuidado de la salud mental de personas mayores en contextos migratorios.

Es importante mencionar que de manera adicional a los artículos científicos, se consultaron libros, capítulos de libros y tesis de grado de autores especialistas en alguno de los elementos de esta triada; para el envejecimiento, se privilegiaron autoras como Dulcey Ruiz en relación con aspectos conceptuales sobre vejez y envejecimiento ${ }^{2}$ y Sandra Huenchuan con sus trabajos sobre derechos humanos y personas mayores ${ }^{3}$; en el eje de salud mental, la tesis de grado de Lopera -Echavarría ${ }^{4}$ y las aproximaciones de Carol Ryff sobre bienestar ${ }^{5}$; en el área de migración, los aportes de Verónica Montes de Oca fueron fundamentales, dado que además de sus abordajes migratorios, ella entrelaza esta situación con variables demográficas como el envejecimiento ${ }^{6}$; estos textos fueron clave para orientar y ampliar la búsqueda sobre la producción individual de cada uno de los autores, con el objetivo de tener un mayor acercamiento a los temas. En lo que respecta a la disciplina de enfermería, la producción es más limitada; se recuperaron para esta revisión los trabajos de Rosimere Santana, enfermera brasileira.

\section{Desarrollo}

Vejez y envejecimiento

Vejez y envejecimiento, si bien están relacionados, corresponden a dos nociones diferenciadas. La primera hace alusión a una etapa del ciclo vital; desde la antigüedad clásica Aristóteles y Platón consideraban la vejez como la última etapa de la vida, para Platón es el momento en que la persona alcanzaba la máxima potencia, sagacidad y juicio; mientras para Aristóteles equivalía al deterioro, ruina y enfermedad. Posterior a ellos y de manera más contemporánea en el siglo XX, se asume con mayor relevancia esta última postura, al considerar la vejez como una etapa de declive biológico ${ }^{7-10}$. El término envejecimiento, por su parte, se entiende como un proceso, que involucra aspectos biológicos, psicológicos y sociales, que presenta análisis teóricos que van desde visiones más fragmentadas, hasta otros con mayores alcances integrativos.

Con énfasis en los aspectos biológicos, se encuentran una serie de teorías que según Goldstein ${ }^{11}$ están divididas en dos grandes grupos: las teorías estocásticas, que afirman que el proceso de envejecimiento sería el resultado de la suma de alteraciones que ocurren en forma aleatoria y se acumulan a lo largo del tiempo, y las teorías no estocásticas que suponen que el envejecimiento estaría predeterminado biológicamente ${ }^{11}$. Según este último, las personas están programadas para envejecer de determinada manera y poco puede hacerse para retardar un proceso que es eminentemente fisiológico ${ }^{12}$; por lo tanto, se entiende desde allí el envejecimiento como una pérdida de funcionalidad que lleva progresivamente a un estado de dependencia y a una limitación para el desarrollo de las actividades básicas que debe realizar la persona mayor.

Desde el enfoque psicológico, la unidad de análisis es el individuo y su forma de encarar el paso del tiempo, por lo que la definición se orienta a la manera cómo la persona enfrenta y responde a las condiciones personales que le toca vivir, a buscar solución a los problemas que tiene, a aceptar y asumir las pérdidas inevitables, para seguir sintiéndose satisfecho e interesado con su existencia ${ }^{13}$. Dentro de este enfoque psicológico, se encuentra la propuesta de envejecimiento exitoso que propone Rowe, en el cual se consideran tres condiciones: una reducida probabilidad de desarrollar enfermedades y discapacidades asociadas, el mantenimiento de un alto nivel de funcionamiento cognoscitivo y fisiológico, así como la participación en actividades sociales y constructivas ${ }^{14}$. En esta misma línea la Organización Mundial de la Salud (OMS), nombra como envejecimiento saludable, al proceso de fomentar y mantener la capacidad funcional que permite el bienestar en la vejez ${ }^{15}$.

Desde el enfoque social, anclado con la sociología, se plantean tres aproximaciones: la teoría funcionalista sobre el envejecimiento, la economía política del envejecimiento y la teoría de la dependencia estructurada; en estas teorías se considera al envejecimiento como el proceso de cambio a lo largo de 
la vida, especialmente en la edad avanzada y la edad como estructura social del fenómeno del envejecimiento; en la primera, se considera la vejez como un quiebre social a partir de la pérdida progresiva de funciones de la persona; la segunda aproximación se encamina a la distribución de los recursos, la situación laboral es el factor detonante de la condición de los adultos mayores al momento de la jubilación; y finalmente, la teoría de la dependencia estructurada propone que la persona debe conservar alguna actividad similar a la que realizaba en su etapa productiva, pues la dependencia se centra en la falta de producción ${ }^{13}$.

Dulcey-Ruiz ${ }^{2}$ y Huenchuan ${ }^{13}$ señalan que el envejecimiento, más allá de un proceso biológico, es un proceso personal de adaptación a los cambios que ocurren tanto en el propio organismo como en el entorno social, por tanto es un proceso que se experimenta de manera diferente, donde algunos pueden generar una adaptación positiva y otros no, en relación con la forma diferencial de afrontar el paso del tiempo y la manera cómo el contexto social integra a las personas mayores.

El termino envejecimiento ha venido adoptando distintos calificativos, como envejecimiento activo, término acuñado por la OMS para describir un proceso donde convergen cuestiones de salud, participación y seguridad, con el fin de mejorar la calidad de vida de la persona a medida que envejece ${ }^{15}$; envejecimiento productivo que se orienta hacia la realización de actividades, bienes o servicios sin importar si reciben o no alguna remuneración económica ${ }^{16}$; envejecimiento saludable, término que acuña la Organización Panamericana de la Salud (OPS). dirigido hacia el fomento y mantenimiento de la capacidad funcional para alcanzar el bienestar en la etapa de la vejez ${ }^{17}$; envejecimiento óptimo que describe Rowe $^{14}$ el cual apunta a envejecer en las mejores condiciones posibles a nivel físico, psicológico y social; y finalmente, envejecimiento positivo, muy similar a la propuesta de la OPS, pues también está bajo el marco de la autosuficiencia y el bienestar como fin ${ }^{18}$.

Estas propuestas ${ }^{14-18}$ a pesar de sustentarse en perspectivas psicosociales, siguen teniendo una fuerte impronta funcionalista que deja de lado una mirada más profunda y critica de los asuntos emocionalesrelacionales inmersos en un proceso, como muestra la producción académica, supera su exclusiva connotación biológica. La lógica más extendida asocia el envejecimiento a una disminución progresiva de la capacidad orgánica y física; relacionándola con enfermedad y deterioro, en una visión homogeneizadora de la experiencia particular de ser una persona vieja. Sin embargo, más allá de su significación biológica, envejecer significa también reconocer, que las trayectorias de vida de las personas se construyen en la interacción social, se debe, además, tomar en consideración los entornos socioculturales, así como las experiencias y los significados que dotan de un sentido singular este proceso.

Dulcey-Ruiz ${ }^{2}$, señala que el envejecimiento equivale al proceso del mismo vivir en el que se conjugan múltiples aspectos y su significado varía de acuerdo a la connotación que cada cultura haga de él, la cultura se entiende como los contextos en los cuales las personas interactúan y se comunican ${ }^{19}$. El envejecimiento cobra un significado, no solo biológico, sino que se asocia a las condiciones en las que se vive a lo largo de la vida: contextos físicos, geográficos, sociales, económicos y culturales, que configuran modos de vida particulares a fin de organizar la experiencia social e individual ${ }^{20}$ del vivir-envejecer de las personas.

\section{Envejecimiento y migración}

Según la Real Academia Española ${ }^{21}$ la migración se define como el desplazamiento geográfico de individuos o grupos, generalmente, por causas económicas o sociales, que constituyen un fenómeno que involucra una dimensión temporal y una dimensión geográfica, en relación con dos lugares: aquel donde se inicia el desplazamiento-región de origen y aquel donde finaliza-región de destino ${ }^{22}$.

Según estudios realizados en México, el patrón identificado consiste en el rejuvenecimiento de las zonas de destino, por emigración de población en edades económicamente productivas, con el correspondiente envejecimiento de las zonas de origen, donde se quedan de manera especial los adultos mayores ${ }^{23,24}$. Estas aproximaciones van mostrando la complejidad del contexto en el que transcurre el proceso de 
envejecimiento en México, situación marcada en algunas regiones que tienen una dinámica migratoria intensa, frecuente e histórica, donde las personas mayores se quedan solas, incluso como responsables del cuidado de otros miembros de sus grupos familiares ${ }^{25,26}$. Si bien los estudios privilegian los análisis de los procesos migratorios de orden internacional, en general en los países latinoamericanos también se comparten patrones de movilidad interna, sobre todo entre lo rural y lo urbano, que también sigue estos perfiles.

En los estudios relacionados con las dinámicas migratorias y el envejecimiento se ha explorado, entre otros asuntos, el impacto que ocasiona el proceso migratorio en aquellos que se van y que después de un tiempo regresan a sus lugares de origen ${ }^{27-30}$; y las afectaciones en la salud mental por motivos de desplazamiento forzado, refugio o por la salida en búsqueda de un mejor nivel económico ${ }^{31,32}$. Se ha encontrado que las personas deciden migrar de sus comunidades de origen porque otros miembros de sus redes familiares o sociales lo han hecho con anterioridad, bajo un patrón denominado migración en cadena $^{33}$, de tal manera que la migración se considera como un hecho social que exige poner en marcha estrategias de comunicación y memoria, para mantener los vínculos entre los que se van y los que se quedan ${ }^{34}$.

Como ya se había señalado, las personas envejecen según la influencia de los factores históricos y socioculturales que definen los estilos y modos de vida ${ }^{32,35,36}$, por lo cual la migración se torna en un problema emergente que amerita ser estudiado, debido a los impactos que ocasiona a nivel social y económico, tanto para los que se han ido, como para aquellos que han regresado a sus lugares de origen $^{28,30,37,38}$.

La migración, realizada por motivos de desplazamiento, refugio o por necesidad económica repercute en la salud mental de los migrantes, pues genera ambivalencia para la toma de decisiones por el choque cultural al que se enfrentan, presencia de estrés postraumático por las condiciones en las que emigran y discriminación por parte de los grupos sociales receptores. Igualmente, estas implicaciones se extienden hacia aquellos que se quedan; Jian-fei ${ }^{39}$ en un estudio de tipo cuantitativo sobre la calidad de vida de los adultos mayores que se quedan cuando los familiares emigran fuera de las zonas rurales de China, encontró que la salud mental estaba significativamente afectada, en comparación con los datos estándar del país, se utilizó una escala de valoración en la cual variables como la edad, el tipo de residencia, la morbilidad, el género y el apoyo económico fueron los principales factores que influenciaron este resultado.

En lo que respecta al contexto migratorio mexicano, son relevantes los estudios de la socióloga Montes de Oca, quien enfatiza cómo la situación migratoria es cada vez más recurrente dada la vulnerabilidad económica que rodea a la población campesina ${ }^{27}$ y que hace que aquella sea la mejor alternativa, lo que conlleva a nuevas dinámicas de funcionamiento familiar y comunitario ${ }^{25,35,40}$ expresado en reajustes en los patrones de apoyo y cuidados hacia los niños y los ancianos ${ }^{25}$.

Tanto Alvarado ${ }^{38}$ como Montes de Oca ${ }^{35}$ muestran que en su gran mayoría son las mujeres las que se quedan al cuidado de los hijos o nietos como resultado del proceso migratorio; que además viene asociado a viudez y otras perdidas, que tienen efectos sobre la salud mental ${ }^{27}$. En un estudio realizado con adultos mayores que migraron hacia Estados Unidos y que posteriormente regresaron a México, se encuentra que estos tienen mayor tendencia a padecer trastornos mentales ${ }^{41}$ mientras que, paradójicamente, otros estudios resaltan el papel de la dinámica migratoria en el fortalecimiento de las redes de apoyo, que impacta positivamente nuevas formas de socialización, nuevos roles al interior de las familias, con lo que se mejora la calidad de vida ${ }^{29}$.

En los estudios realizados con adultos mayores que se quedan en México, con descendencia tanto en este como en Estados Unidos, se encuentra que la decisión de quedarse obedece al apego a la tierra, la nostalgia por el hogar, las dificultades de la movilidad a Estados Unidos y la existencia de otros miembros de la familia que no han migrado; a la par que manifiestan sentimientos de tristeza, angustia o ansiedad ante la posibilidad de saber que sus hijos han muerto en los Estados Unidos, estén presos o no han 
logrado concluir el tránsito exitosamente ${ }^{41}$. Otros estudios como los de Mayston et al ${ }^{42}$ y Grijalva-Monteverde $^{43}$, realizados con población Mexicana, Peruana, China y Nigeriana, resaltan el papel significativo de las remesas en la mejora de la calidad de vida de los adultos mayores.

A nivel familiar, se documentó que la migración no sólo trastoca la estructura y composición de los hogares, sino que también contribuye a cambiar la vida cotidiana de los que se quedan, ya sea que se propicie una redefinición de roles o se introduzcan cambios en las actividades que realizan. Paradójicamente, se extraña al que emigró, pero se reconoce su papel en la solvencia económica de la familia; en tanto otros ven este proceso de migración como una amenaza que genera perdida de la confianza, conflictos familiares y/o rupturas conyugales. Los que se quedan utilizan procesos defensivos que les permiten disminuir la desorganización familiar, el dolor y la frustración, lo que genera un ambiente ambivalente, dada la esperanza sobre el posible retorno del que se fue ${ }^{44}$.

Ramírez-Contreras ${ }^{45}$ psicóloga mexicana, se ha acercado a estos fenómenos desde su propuesta de cuidado trasnacional en familias trasnacionales; de acuerdo a este término de Bryceson y Vuorela, para referirse a familias que se conforman con miembros que viven una o la mayor parte del tiempo separados los unos de los otros, que son capaces de crear vínculos que permiten que sus miembros se sientan parte de una sola unidad y perciban su bienestar desde una visión colectiva a pesar de la distancia física, dándole un especial valor al apoyo emocional como parte del cuidado trasnacional de las personas mayores.

\section{Envejecimiento y salud mental}

En los contextos migratorios, el proceso de envejecimiento y los aspectos emocionales y relacionales, adquieren especial resonancia, con su correspondiente articulación al campo de la salud mental. El concepto oficial de salud mental lo propuso la OMS en 1950; definiéndola como: Una condición sometida a fluctuaciones debido a factores biológicos y sociales, que permite al individuo alcanzar una síntesis satisfactoria de sus propios instintos, potencialmente conflictivos; formar y mantener relaciones armónicas con terceros y participar en cambios constructivos en su entorno social y físico ${ }^{46}$. Esta definición, sigue vigente, aunque en una versión abreviada: estado de bienestar en el cual el individuo es consciente de sus propias capacidades, puede afrontar las tensiones normales de la vida, puede trabajar de forma productiva y fructífera y es capaz de hacer una contribución a su comunidad ${ }^{47}$.

En el momento de su promulgación, la OMS dio respuesta a intereses políticos y sociales para preservar a la humanidad de nuevas guerras, por lo que se hacía deseable la armonía entre los hombres, la armonía consigo mismo y una relación con el entorno, de esta manera se infiere que el concepto que se propuso se relacionó en forma importante con la finalización de la guerra y sus consecuencias; es importante señalar que también refleja la idea que la salud mental no debe considerarse como algo independiente de la salud general, ya que implica una concepción integral de la persona, que abarca aspectos físicos, psicológicos y sociales de manera interrelacionada. A pesar de que esta definición asume una postura decidida hacia el ámbito de las relaciones, su operativización ha tomado distintos rumbos.

Uno de ellos, ha sido la exploración de la salud mental en relación con la ausencia de enfermedad, como un criterio de normalidad, cuyo supuesto ontológico es que lo mental se equipare a procesos de orden biológico; desde esta perspectiva, la salud mental se expresa paradójicamente a partir de la enfermedad o el trastorno mental, los análisis epidemiológicos y las propuestas farmacológicas. Las tendencias de los estudios en salud mental de los adultos mayores, se concentran en problemas como la depresión $^{48,49}$ o la prevención del suicidio ${ }^{50}$.

Otra de las vías, comúnmente encontrada en la literatura es la que asocia salud mental con el término de bienestar, el cual es abordado como un componente vital en la vida del ser humano; a partir de dos orientaciones filosóficas: el hedonismo y la eudaimonia. Para el hedonismo, cuyo principal representante es Epicuro; el bienestar equivale a experimentar la mayor cantidad posible de placer ${ }^{51}$; esta postura da como resultado conceptos como el de bienestar subjetivo para referirse al balance afectivo y la satisfacción vital percibida ${ }^{52}$. La segunda corriente eudaimónica, con Aristóteles entre sus pensadores, establece

Envejecimiento y salud mental en contextos migratorios • E. Torres-Hernández, B. Arias-López 
que el bienestar se refiere a vivir de forma plena o dar realización a los potenciales humanos más valio$\operatorname{sos}^{53}$, esto es, la realización de actividades congruentes, con valores profundos y con compromiso pleno, que permite que las personas se sientan vivas y auténticas ${ }^{54}$.

Carol Ryff ${ }^{55}$, toma elementos de esta segunda orientación para proponer el término de bienestar psicológico definiéndolo como el desarrollo del verdadero potencial de la misma persona, por lo que alcanzar el bienestar psicológico sería el resultado de una vida bien vivida; esta propuesta se concreta en un instrumento de medición diseñado por la autora ${ }^{56}$, que engloba seis dimensiones: auto aceptación, relaciones positivas con los demás, dominio del ambiente, autonomía, crecimiento personal y propósito en la vida, las cuales se integran en la llamada Escala de Bienestar Psicológico.

Finalmente, otros autores han desarrollado la noción de bienestar social, para incorporar también lo social, el mundo intersubjetivamente construido, y la historia ${ }^{57}$, es decir, integrar las circunstancias y el funcionamiento de la sociedad a las experiencias subjetivas y sus representaciones culturales ${ }^{58}$.

En estos desarrollos, salud mental y bienestar parecen equipararse, ya que incorporan una perspectiva subjetiva, psicológica y social. La salud mental trata sobre el cómo y por qué las personas experimentan su vida de forma positiva e incluye una dimensión cognitiva y otra afectiva; el bienestar se centra en el desarrollo personal, en el estilo y manera de afrontar los retos vitales y el esfuerzo por conseguir las $\operatorname{metas}^{59-61}$.

En los estudios en enfermería sobre envejecimiento, la noción de bienestar acompaña propuestas como las del envejecimiento deseado, exitoso o funcional, en relación con la búsqueda de otra dimensión del vivir; a través de las decisiones positivas que debe tomar la persona mayor, además de utilizar su potencial humano, para reaccionar a las adversidades que el envejecimiento puede provocar ${ }^{62}$. Ferreira y dos Santos ${ }^{63}$ afirman que las personas mayores, en el continuo de la vida, no quieren ser viejas (vivir lo indeseado con malestar) ... ellas quieren tornarse ancianas (envejecer con bienestar).

Bajo estas perspectivas encuentra resonancia la propuesta de Lopera ${ }^{64}$, quien incorpora el termino de salud mental como sabiduría práctica para referirse a un saber vivir la vida que no puede ser aprendido mediante libros o transmisión teórica, sino en la vida misma asumiendo la responsabilidad de las propias decisiones; esta propuesta está basada en los planteamientos que hace Canguilheim sobre lo normal, definiéndolo como la capacidad de instituir nuevas normas de vida, mientras que lo patológico es el apego a normas caducas, que no permiten la creación de otras que sean adecuadas al medio físico y social ${ }^{65}$; por lo tanto, ser saludable es vivir conforme a la propia norma, aunque el devenir pueda llevar al cambio de dicha norma para poder afrontar mejor las variaciones del ambiente ${ }^{64}$. Bajo este argumento filosófico, la salud mental estará presente cuando existe armonía entre las propias tendencias, motivaciones, razones, valores, creencias con las del otro como un compañero en la existencia.

\section{Conclusión}

Realizar esta revisión, permitió encontrar un acervo investigativo importante en relación con el proceso de envejecimiento y su relación con la salud mental, en contextos migratorios. La tendencia mayor es el acento en los análisis funcionalistas expresados en distintos patrones de morbilidad, de manera especial, centrados en lo biológico, y en pocas ocasiones situados en las condiciones sociales, políticas y económicas. Este ámbito, amerita una perspectiva de la salud mental que interrogue la manera cómo se tramitan las situaciones derivadas de los conflictos que deben resolverse en la vida cotidiana y que impone la vida social, donde la dinámica o contexto migratorio, interés que nos ocupa, ha venido marcando de manera particular a la población mexicana, especialmente a los adultos mayores que han vivenciado la partida de sus familiares con el objetivo de tener una mejor vida.

Si bien el proceso de envejecimiento ha cobrado gran relevancia en el mundo y en el contexto latinoamericano, posicionándose como un tema clave de análisis y reflexión; el reto para la disciplina de enfermería es dirigir la mirada a situaciones que han permanecido invisibles en la generación de 
conocimiento y desarrollo de prácticas de cuidado, como es el caso de la migración, la cual se sitúa como uno de los desafíos de la salud a nivel mundial y su relación con la atención y el cuidado.

Esta situación se identifica como necesidad investigativa por disciplinas como la psicología, la sociología, la antropología, entre otras; pero en lo que respecta a la disciplina enfermera, apenas se esboza el interés por poner en dialogo el cuidado de adultos mayores en situaciones migratorias como las descritas. La migración, con las adaptaciones que conlleva para la población adulta mayor que se queda en sus lugares de origen, exige de los profesionales de enfermería generar una mejor comprensión y conocimiento del adulto mayor y la red social con la que cuenta, para reconocer los efectos que ha tenido sobre las trayectorias personales y sociales, la interacción que tienen con su entorno, la forma en que resuelven las situaciones que se presentan en el día a día, en pocas palabras, conocer las prácticas que se gestan para resolver su vida cotidiana en ausencia de los familiares que han migrado.

Explorar la triada envejecimiento/salud mental/migración le permitirá a la enfermería aportar respuestas concretas a situaciones contextuales en donde interaccionan los adultos mayores, desde aquello que piensan y sienten la personas, desde lo qué hacen y desde sus propios saberes, esto es, aportar cuidados culturalmente situados para el logro del bienestar.

\section{Responsabilidades éticas}

Protección de personas y animales: Las autoras declaran que para esta investigación no se han realizado experimentos en seres humanos ni en animales, ya que se trata de una investigación documental.

Confidencialidad de los datos: Las autoras declaran que en este artículo no aparecen datos de participantes.

Financiamiento: No se recibió patrocinio para llevar a cabo esta investigación.

Conflicto de intereses: Los autores declaran no tener conflicto de intereses.

\section{Referencias}

1. Silamani J, Guirao G. Utilidad y tipos de revisión de literatura. ENE, Rev Enfermería 2015;9(2):1-16. [Consultado agosto 16 2017]. Disponible en: https://bit.ly/2mYZnuY

2. Dulcey-Ruiz E. Envejecimiento yVejez. Categorías y Conceptos. Bogotá Colombia. Red Latinoamericana de Gerontología; 2015. 587 p.

3. Huenchuan S. Los derechos de las personas mayores. Bol Nac Unidas 2011;16. [Consultado febrero 24 2016]. Disponible en: https://bit.ly/2Fzb2tm

4. Lopera-Echavarría JD. Sabiduría práctica y salud psíquica. [Tesis doctoral]. Colombia: Facultad de Ciencias Sociales y Humanas. Universidad de Antioquia; 2014.

5. Ryff C, Keyes C. The Structure of Psychological Well-Being Revisited. J Pers Soc Psychol. 1995;69(4):719-27.

6. Montes de Oca V. Envejecimiento en America Latina y el Caribe. México: Instituto de Investigaciones Sociales UNAM. 2013.672 p.

7. Ramos-Esquivel J, Meza-Calleja AM, Maldonado-Hernández I, Ortega-Medellín MP, Hernández-Paz MT. Aportes para una conceptualización de la vejez. Rev Educ Desarro. 2009;(11):47-56.

8. Jorquera P. Vejez y envejecimiento: Imaginarios sociales presentes en los textos escolares oficiales del Ministerio de educación chileno. Rev MAD. 2010; 22:132-65.

9. Gómez-Cabrera AP. Una mirada de desolación. El estigma y el abandono en la vejez. Calid. vida salud. 2013;6(2):91-9.

10. Carbajo-Vélez M. La historia de la vejez. Ensayos 2008(18):237-54. [Consultado febrero 24 2016]. Disponible en: https://bit.ly/2W1aJwq

11. Goldstein S. The biology of aging. N Engl J Med. 1971;(285):1120-9.

12. Sarabia-Cobo CM. Envejecimiento exitoso y calidad de vida: Su papel en las teorías del envejecimiento. Gerokomos 2009; 20(4):172-4. [Consultado marzo 2 2016]. Disponible en: https://bit.ly/2RAFUAc 
13. Huenchuán S, Rodríguez-Piñero Royo L. Envejecimiento y derechos humanos: situación y perspectivas de protección. New York: CEPAL 2010;144.

14. Rowe, JW, Kahn RL. Successful Aging. Gerontologist. 1997;37 (4):433-40.

15. Organización Mundial de la Salud (OMS). Informe mundial sobre el envejecimiento y la salud. Ginebra: OMS; 2015.

16. Miralles I. Envejecimiento Productivo: Las contribuciones de las personas mayores desde la cotidianidad. Trab y Soc. 2011; XV (16):137-61. [Consultado marzo 2 2016]. Disponible en: https://bit.ly/2HqwtyB

17. Organización Panamericana de la Salud. Curso de vida saludable: Envejecimiento Saludable. Washington: OPS; 2011 p. 1-6. [Consultado febrero 18 2014]. Disponible en: https://bit.ly/2CxJmBa

18. Calvo E. Envejecimiento Positivo. En: Diplomado de Gerontología Social. Chile: Pontificia Universidad Católica; 2013. p. 47-62. [Consultado marzo 2 2016]. Disponible en: https://bit.ly/2RKrzRS

19. Alvarado-García AM, Salazar-Maya AM. Análisis del concepto de envejecimiento. Gerokomos. 2014; 25(2):57-62.

20. Martino M. Modos de vida: debates y aportes para el trabajo social con familias. Rev Textos Context Porto Alegre. 2009; 8(1):3-21.

21. Real Academia Española. Diccionario de la lengua española. Madrid: RAE; 2016. [Consultado junio 6 2016]. Disponible en: http://dle.rae.es/?id=PE38JXc

22. Welti C. Demografía I. México, DF: PROLAP; 1997. 97-121 p.

23. Consejo Nacional de Población. Indice Absoluto de Intensidad Migratoria México-Estados Unidos 2010. México: CONAPO; 2010. [Consultado marzo 22 2016]. Disponible en: https://bit.ly/2MnIN1d

24. Consejo Nacional de Población. Anuario de migración y remesas. 2014.México: CONAPO/Fundación BBUV Bancomer; 2014. 1-164 p.

25. Montes de Oca-Zavala V, Sáenz R, Santillanes-Allende NI, Izazola-Conde C. Cuidado a la salud en la vejez y recursos familiares transnacionales en México y Estados Unidos. Uaricha Rev Psicol. 2012;9(19):85-101.

26. Montes de Oca-Zavala V, Hebrero M. Dinámica familiar, envejecimiento y deterioro funcional en México. Kairós. 2008;11(1):143-66.

27. Montes de Oca-Zavala V, Ramírez- García T, Sáenz R, Guillen J.The Linkage of Life Course, Migration, Health, and Aging: Health in Adults and Elderly Mexican Migrants. J Aging Health. 2011;23(7):111640. https://doi.org/10.1177/0898264311422099

28. Vilar Peyrí E, Eibenschutz-Hartman C. Migración y Salud mental: un problema emergente de salud pública. Rev Gerenc y Política Salud. 2007;6(13):11-32. [Consultado febrero 24 2016]. Disponible en: https://bit.ly/2zolRu3

29. Marques FD, Sousa L. Trajetórias e vida de pessoas idosas ex-emigrantes Portugueses: a construção da integridade familiar. Rev Kairós 2011;14(4):3-24.

30. Jansà JM. Inmigración y envejecimiento, nuevos retos en salud pública. Gac Sanit. 2006; 20 (supl 1):10-4. [Consultado marzo 6 2016]. Disponible en: https://bit.ly/2AT5UME

31. Norambuena-Coloma K, Mendoza-Parra S. Los desafíos en salud del migrante para la Enfermería profesional. Enfermería Glob. 2008; 14:1-8.

32. Barbosa de Campos M, Flávio-Barbieri A. Considerações teóricas sobre as migrações de idosos. Rev Bras Estud Popul. 2013;30:S69-84.

http://dx.doi.org/10.1590/S0102-30982013000400005

33. Martínez-Nava A, Muñoz-Güemes A. Estudio de caso de dos comunidades con migración indígena en el municipio de Tamuín, S.L.P. Tlatemoani. 2010;(4):1-18. [Consultado marzo 8 2016]. Disponible en: https://bit.ly/2AY2Ejg 
34. Hernández Almazán JM. “¿Ya se olvidaron de nosotros? La construcción social de la memoria en torno a las fotografías de migrantes y difuntos. Santa María del Río, S.L.P.” [Tesis de maestría]. San Luis Potosí: El Colegio de San Luis, AC; 2012.

35. Montes de Oca-Zavala V. Viudez, soledad y sexualidad en la vejez: mecanismos de afrontamiento y superación. Rev Kairós. 2011; 14(5):73-107.

36. Iorio-Rates M, Lopes A. Envelhecimento, cultura e os judeus poloneses no Brasil. Rev Kairós. 2013;16(5):165-88.

37. Franken I, de Lima-Coutinho M, Pereira-Ramos MN. Representações sociais, saúde mental e imigração internacional. Psicol Ciênc Prof. 2012; 32(1):202-19.

http://dx.doi.org/10.1590/S1414-98932012000100015

38. Alvarado R. Salud mental en inmigrantes. Rev Chil Salud Pública. 2008;12(1):37-41.

39. Jian-fei X, Si-qing D, Zhu-qing Z, Qi-feng Y, Sai-nan Z, Jun-hua H. et al. Mental health is the most important factor influencing quality of life in elderly left behind when families migrate out of rural China. Rev Lat Am Enfermagem 2014; 22(3):364-70. http://dx.doi.org/10.1590/0104-1169.3400.2425

40. Montes de Oca- Zavala V. La discriminación hacia la vejez en la ciudad de México: contrastes sociopolíticos y jurídicos a nivel nacional y local. Perspectivas sociales 2013;15(1):1-34.

41. Montes de Oca-Zavala V, Díaz-Ábrego M, Hebrero-Martínez M. Migración, salud y masculinidad. Don Leovigildo y su familia: tres generaciones entrelazadas por la salud y migración en Guanajuato. Estudio de caso. Rev del Cent Investig (Méx). 2012; 10 (38):85-101.

42. Mayston R, Guerra M, Huang Y, Sosa AL, Uwakwe R, Acosta I, et al. Exploring the economic and social effects of care dependence in later life: protocol for the 10/66 research group INDEP study. Springerplus. 2014;3(1):379. https://doi.org/10.1186/2193-1801-3-379

43. Grijalva-Monteverde G, Zúñiga-Elizalde M, Zupo-Jiménez MJ. Adultas y adultos mayores en Sonora: ¿dependientes, autosuficientes o proveedores? Región y Soc. 2007;19( ${ }^{\circ}$ spe):117-45. [Consultado febrero 24 2016]. Disponible en: https://bit.ly/2RVbS9F

44. Hurtado-Arriaga G, Rodríguez-Contreras V, Escobar-Torres J, Santamaría Suárez S, Pimentel-Pérez B. “Los que se quedan” Una experiencia de migrantes. Rev científica electrónica Psicol. 2010;(6): 9-28. [Consultado febrero 24 2016]. Disponible en: https://bit.ly/2CBVelK

45. Ramírez-Contreras MG. Tecnologías de la comunicación y emocionalidad en familias transnacionales. Rev. Inter Psicol 2014;13(2):1-46. [Consultado Mayo 15 2016]. Disponible en: https://bit.ly/2FN1x9k

46. Bertolote J. Raíces del concepto de salud mental. World Psychiatry. 2008;6(2):113-6.

47. Organización Mundial de la Salud [OMS]. Promoción de la Salud Mental. Conceptos. Evidencia emergente. Práctica: Informe Compendiado. Ginebra: OMS; 2004. [Consultado marzo 8 2016]. Disponible en: https://bit.ly/2FDXY5S

48. Aguilar-Navarro S, Ávila-Funes J. La depresión: particularidades clínicas y consecuencias en el adulto mayor. Gac Méd Mex 2007;143(2):141-8. [Consultado febrero 24 2016]. Disponible en: https://bit.ly/2HqdDaF

49. Belló M, Puentes-Rosas E, Medina-Mora ME; Lozano R. Prevalencia y diagnóstico de depresión en población adulta en México. Salud Publica Mex. 2005;47 supl I: S4-S11. [Consultado febrero 24 2016]. Disponible en: https://bit.ly/2FRgkj8

50. Ribot-Reyes V, Alfonso-Romero M, Ramos-Arteaga ME, González-Castillo A. Suicidio en el adulto mayor. Rev haban cienc méd. 2012;11(5):699-708. [Consultado febrero 24 2016]. Disponible en: https://bit.ly/2T8AVTY

51. Diener E, Suh EM, Lucas RE, Smith HL. Subjective well-being: three decades of progress. Psychol Bull. 1999;125(2):276-302. http://dx.doi.org/10.1037/0033-2909.125.2.276

52. Lucas RE, Diener E, Suh E. Discriminant Validity of Well-Being Measures. J Pers Soc Psychol. 1996;71(3):616-28. http://dx.doi.org/10.1037/0022-3514.71.3.616 
53. Ryan R, Huata V, Deci E. Living well: a self-determination theory persepctive on eudaimonia. J Happines Stud. 2008;9:139-70. http://dx.doi.org/10.1007/s10902-006-9023-4

54. Waterman AS. Two Conceptions of Happiness: Contrasts of Personal Expressiveness (Eudaimonia) and Hedonic Enjoyment. J Personal Soc Psychol. 1993; 64(4):678-91. http://dx.doi.org/10.1037/0022-3514.64.4.678

55. Ryff C. Happiness is everything, or is it? explorations on the meaning of psychological well-being. J Personal Soc Psychol 1989; 57(6):1069-81. http://dx.doi.org/10.1037/0022-3514.57.6.1069

56. Díaz D, Rodríguez-Carvajal R, Blanco A, Moreno-Jiménez B, Gallardo I, Valle C, et al. Adaptación española de las escalas de bienestar psicológico de Ryff. Psicothema. 2006;18(3):572-7.

57. Blanco A, Díaz D. El bienestar social: su concepto y medición. Psicothema. 2005;17 (4):582-9.

58. De Almeida-Filho N, Ávila-Coelho MT, Tourinho-Peres MF. O Conceito de Saúde Mental. Rev USP. 1999; 43:100-25.

59. Blanco A, Díaz D. Orden social y salud mental: Una aproximación desde el bienestar social. Clínica y Salud. 2006;17(1):7-29.

60. Díaz-Llanes G. El bienestar subjetivo. Actualidad y perspectivas. Rev Cuba Med Gen Integr. 2001;17(6):572-9.

61. Vázquez C, Hervás G, Rahona JJ, Gómez D. Bienestar psicológico y salud: aportaciones desde la psicología positiva. Anu Psicol Clín Salud. 2009;5(1):15-28.

62. Dos Santos I, Dos Santos-Alves A, Leandro da Silva AF, Pereira-Caldas C, Miguéis-Berardinelli LM, Ferreria-Santana R. O grupo pesquisador construindo ações de autocuidado para o envelhecimento saudável: pesquisa sociopoética. Esc Anna Nery. 2011;15(4):746-54. http://dx.doi.org/10.1590/S1414-81452011000400013

63. Ferreira-Santana R, Dos Santos I. Como Tornar-Se Idoso: Um Modelo De Cuidar Em Enfermagem Gerontológica. Texto contexto enferm 2005; 14(2):202-12.

http://dx.doi.org/10.1590/S0104-07072005000200007

64. Lopera-Echavarría JD. Salud mental y sabiduría práctica. Un intento de integración y aproximación conceptual. Tesis Psicológica. 2012;(7):60-75.

65. Canguilhem G. Lo normal y lo patológico. México: Siglo XXI; 1984. 